\title{
GW23-e0475 EFFECTS OF PERITONEAL COOLING ON INFLAMMATION AFTER CARDIOPULMONARY RESUSCITATION IN RABBITS
}

doi:10.1136/heartjnl-2012-302920a.119

HongYan Wei, Xin Li, XiaoXing Liao, Xuan Dai, Rong Liu, YingQing Li, ChunLin Hu. Department of Emergency Medicine, the First Affiliated Hospital of Sun Yat-Sen University, Guangzhou, 510080, China

Objectives To explore the effects of different cooling methods on systemic inflammation after cardiopulmonary resuscitation (CPR) in New Zealand rabbits.

Methods Forty eight adult New Zealand rabbits were induced ventricular fibrillation by AC current and were resuscitated after cardiac arrest for $5 \mathrm{~min}$. After restore of spontaneous circulation (ROSC), the rabbits were randomly divided into four groups according to the way of cooling methods, nomothermia group (NT), peritoneal cooling group (PC), surface cooling group(SC) and local cooling group(LC). The plasma concentration changes of tumour necrosis factor- $\alpha$ (TNF- $\alpha$ ) and interleukin-6 (IL-6)were measured in each group at different time points before and after ROSC. Liver tissue were removed after ROSC $12 \mathrm{~h}$, the level of nuclear

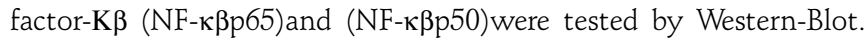
The survival time was recorded and compared after ROSC $96 \mathrm{~h}$. One-way ANOVA or Mann-Whitney rank was used to determine the statistical significance between two groups. LSD-t test for multiple comparisons, $\mathrm{R} \times \mathrm{C}$ test for ROSC comparisons.

Results The levels of plasma TNF-a concentration in PC group were inferior to NT group after ROSC, p value were 0.020, 0.010 and 0.014 at $24 \mathrm{~h}, 48 \mathrm{~h}$ and $72 \mathrm{~h}$ respectively. The TNF-a level in PC group was also inferior to SC and LC group after ROSC $72 \mathrm{~h}$ (PC: $\mathrm{SC}, \mathrm{p}=0.020$; PC: LC, $\mathrm{p}=0.042)$. The IL-6 levels in PC group were inferior to NT group after ROSC $12 \mathrm{~h}$, p value was $0.013,0.03,0.010$ and 0.009 respectively. The concentrations of P65 and P50 in PC group were lower than those in other groups $(p<0.05)$, while there were no differences between the other three groups. The average survival time was $19.5 \mathrm{~h}, 57 \mathrm{~h}, 37.3 \mathrm{~h}, 21.5 \mathrm{~h}$ in NT, PC, SC and LC group after ROSC respectively, (PC:NT, $p=0.024$; PC:SC, $p=0.128$; PC:LC, $\mathrm{p}=0.052$, but SC:NT, $p=0.319$, SC:LC, $p=0.266$ ).

Conclusions The neotype peritoneal cooling could rapidly induce and maintain hypothermia, and decrease the peritoneal temperrature quickly, thus inhibit liver NF- $\kappa \beta$ activation, reduce TNF-a and 


\section{ABSTRACTS}

IL-6 release, subsequently relieve systemic inflammation after ROSC and prolong rabbit survival time. 